\title{
Milk and Peace, Drought and War. Somali Culture, Society and Politics. Essays in Honour of I. M. Lewis.
}

London, Hurst \& Company, 2010, 437 p., bibl.

\section{Alain Gascon}

URL : http://journals.openedition.org/etudesafricaines/18056

DOI : 10.4000/etudesafricaines.18056

ISSN : $1777-5353$

Éditeur

Éditions de l'EHESS

Édition imprimée

Date de publication : 1 avril 2015

ISSN : 0008-0055

\section{Référence électronique}

Alain Gascon, « Milk and Peace, Drought and War. Somali Culture, Society and Politics. Essays in Honour of I. M. Lewis. », Cahiers d'études africaines [En ligne], 217| 2015, mis en ligne le 31 mars 2017, consulté le 24 septembre 2020. URL : http://journals.openedition.org/etudesafricaines/18056 ; DOI : https://doi.org/10.4000/etudesafricaines. 18056

Ce document a été généré automatiquement le 24 septembre 2020.

(C) Cahiers d'Études africaines 


\section{Milk and Peace, Drought and War. Somali Culture, Society and Politics. Essays in Honour of I. M. Lewis.}

London, Hurst \& Company, 2010, 437 p., bibl.

Alain Gascon

HOEHNE, Markus V. \& LULING, Virginia (eds.). - Milk and

Peace, Drought and War. Somali Culture, Society and Politics. Essays in Honour of I. M. Lewis. London, Hurst \& Company, 2010, 437 p., bibl.

Ioan Myrddin Lewis est reconnu par la communauté internationale des somalisants comme le père-fondateur des études somali. Depuis près de soixante ans, il a accompli une œuvre fondamentale tant en anthropologie, qu'en linguistique, qu'en histoire et qu'en science politique. D'abord étudiant en chimie à Glasgow, il suivit ensuite les cours d'Evans-Pritchard à Oxford et travailla, sous la direction de Franz Steiner, à l'International African Institute de Londres où il rédigea le volume sur les Somali, les Afar et les Saho. Ayant appris le somali avec B. W. Andrzejewski, il partit en 1955 pour le protectorat du Somaliland britannique où les autorités le laissèrent mener ses recherches à sa guise. Il assista ainsi aux réunions de la Somali Youth League (SYL), qui demandait l'indépendance, puis au Sud, dans la Somalie sous la tutelle italienne ${ }^{7}$, aux meetings du mouvement fédéraliste Hizbia/Xizbia Digil-Mirifle Somalia (H/XDMS). Il tira de ses travaux un rapport, destiné aux autorités coloniales: "The Somali Lineage System and the Total Genealogy » et l'ouvrage qui fit date, A Pastoral Democracy, et qui lui ouvrit une longue carrière universitaire. Partisan engagé de l'union des Somalies britannique et italienne (1960), il poursuivit et étendit ses études à l'ensemble de la république. En 1969, il accueillit favorablement, comme bien des Somaliens ${ }^{8}$, le coup d'État autoritaire, nationaliste et socialiste du général Maxamed Siyaad Barre qui 
s'attaqua à la corruption et décida d'écrire le somali, langue officielle, en caractères latins. En 1966, l'Unesco l'avait appelé, avec B. W. Andrzejewski et J. Tubiana, comme expert auprès du gouvernement somalien afin de l'aider à choisir entre les caractères « indigènes » arabe et latin pour écrire le somali. Il fut l'un des premiers à alerter l'opinion sur les dérives dictatoriales du régime qui, accentuées après la défaite en Ogadén (1977-1978), aboutirent à la guerre civile au Nord, dès 1988, et la chute de Siyaad en 1991. Depuis plus de vingt ans, l'État somalien, pourtant porté par un vigoureux sentiment national, manifesté par une résistance opiniâtre aux colonisateurs et des traits culturels communs (langue, islam), se déchire entre factions, confréries, seigneurs de la guerre, segments de clans. Bien pis, le Somaliland a rompu l'union et s'est déclaré indépendant dans les limites tracées par le colonisateur'.

2 Les médias, et parfois des chercheurs, ont tiré des études de Lewis sur les clans - qui rendraient les Somaliens rétifs à toute forme d'État - la seule explication du naufrage de l'État somalien. Face à la persistance de cet échec, qui parait remettre en question son engagement et son œuvre, le "père-fondateur» a repris ses travaux ${ }^{10}$ tout en faisant face aux critiques de son «fonctionnalisme». On aurait pu craindre, toutefois, que ce livre rédigé en son honneur ne fit l'impasse sur les débats suscités par ses articles et ses livres même si C. Geshekter intitule le dernier paragraphe de sa conclusion: «Somali Studies 2010 : we are all Lewisites» (p. 398). En effet, quand on referme ce gros ouvrage on doit reconnaître que les somalisants ont été, sont et seront, un jour ou l'autre, « lewisiens »!

3 Rédiger le compte rendu exhaustif d'un tel gros livre est une gageure car les contributeurs envisagent l'ensemble des thèmes abordés par I. M. Lewis tout au long d'une œuvre de plus d'un demi-siècle. Après une introduction rappelant les questions toujours en débat dans les études somaliennes, les deux directeurs de l'ouvrage ont classé les 21 contributions en huit parties d'inégale importance: " 1 . De la période coloniale à aujourd'hui; 2 . La politique clanique, l'économie pastorale et le changement ; 3. L'Islam ; 4. Les possessions ; 5. La poésie ; 6. Les "variations culturelles" (sic.) ; 7. La langue ; 8. Conclusion ». Dans cet ensemble, deux articles sur «la métrique de la poésie somali » et « la structure de la coordination » retiendront l'attention des seuls spécialistes de la linguistique du somali. Cependant, je signale aux lecteurs la contribution, originale et éclairante (pp. 345-363), de M. V. Hoehne (avec Muuse Cali Faruur et Axmed Cabdullahi Du'aale) sur les surnoms en Somalie. Ils décrivent les circonstances de la naissance du bébé, son apparence et son caractère où portent les espoirs de ses parents: ils sont exclusivement en somali alors que les noms font référence, en arabe, à l'islam. Jusque dans leurs patronymes, les Somali montrent leur double fidélité au monde arabo-musulman et à la culture somali.

4 Je me propose d'aborder et de discuter dans l'ensemble des contributions les apports les plus novateurs et les plus pertinents pour les études somali. Tous ces textes ont en commun de remettre en question l'homogénéité de la Somalie, des Somaliens, des Somali et du somali. « Unity in diversity » écrit V. Luling (p. 305).

5 Comme beaucoup de somalisants, j'ai répété et écrit ce que nos maitres, somali et européens, nous ont enseigné : "En somali, il n'y a pas de dialecte $»^{11}$. Quelques variations lexicales régionales, d'ailleurs mineures, n'empêchent pas l'intercompréhension de tous les locuteurs du somali du nord-est du Kenya à Djibouti, de Berbera à Xamar (Mogadiscio) et à Kismaayo. Or, c'est loin d'être le cas comme le démontrent des contributions dont les arguments, incontestables, remettent en 
question l'uniformité linguistique des Somali, notamment «Language Marginalisation, Ethnic Nationalism and Cultural Crisis in Somalia » de Mohamed Haji Mukhtar (pp. 279-300) qui appartient à la partie "Cultural Variations» (sic.). Deux cartes (pp. 299-300), hélas peu lisibles même à la loupe, confirment la division des locuteurs du somali en deux grands groupes ${ }^{12}$. Les Af-Maay (Af-Reewiin, Maaymaay ou Maayteri) prédominent dans les vallées inférieures et moyennes du Wabi Shabeele et du Jubba, dans le nord-est du Kenya, dans le sud-ouest de l'Éthiopie et dans le Benaadir. Le somali officiel (Af-Maxaa ou Af-Soomali) domine au Centre et au Nord et dans des enclaves éparpillées dans la Mésopotamie somalienne, en Ogadén et au nord-est du Kenya (p. 293). Se référant à ses enquêtes et à des travaux récents, l'auteur rappelle que l'AfMaxaa est ressenti comme un idiome étranger par les locuteurs de l'Af-Maay. Les résistances à la campagne d'alphabétisation lancée en 1972 par Siyaad Barre, dénoncées comme «antirévolutionnaires " par ses séides, furent réprimées comme telles par les milices et le parti unique. Les persécutions continuèrent après la chute de la dictature et il fallut attendre la Conférence pour la réconciliation et la paix en Somalie, tenue à Nairobi en 2002, pour que la Charte fédérale de 2003 reconnût : «The official language of the Somali Republic shall be Somali (Maay and Maxaatiri [Af-Maxaa]) » (p. 292). Sans doute, la recherche a-t-elle progressé ces quarante dernières années, mais ce pieux mensonge, cet accommodement avec la réalité, est un chapitre de plus à verser à la maladie infantile du nationalisme qui ne touche pas que l'Afrique.

Je rapprocherai les contributions de V. Luling : «Farmers from Arabia : the Role of Gibil Cad Groups in the Interior of Southern Somalia» (pp. 301-317) et de Ken Menkhaus: "The Question of Ethnicity in Somali Studies : The Case of Somali Bantu Identity (pp. 87-104). Ces auteurs se proclament, en effet, les héritiers d'I. M. Lewis, mais remettent en cause la lecture simplificatrice de son œuvre résumée au titre de la seule « Pastoral Democracy » alors qu'il n'a cessé de publier sur l'ensemble de la Somalie, reprenant ses écrits les plus anciens à la lumière de ses recherches et de l'actualité, comme le fit Paul Pélissier dans Campagnes africaines en devenir ${ }^{13}$. V. Luling et K. Menkhaus ont travaillé parmi les Somaliens, majoritairement agriculteurs et "moins égaux que les autres", des rives du bas-Shabeele et du bas-Jubba auprès desquels Lewis avait mené des études pionnières. En dépit de la construction par les Cad Gelebi de généalogies les rattachant à la péninsule Arabique et de stratégies d'adoption négociées par les «Bantous » auprès de grands clans, ces populations souffrent plus que les autres de la disparition de l'État. Dépossédées par les Italiens lors du développement des périmètres irrigués, elles continuent de perdre leurs terres ou d'être rançonnées par les chefs de guerre car elles n'ont pas de milices à leur disposition. Le plus souvent descendants d'esclaves, on les considère comme des Bantous "étrangers " même s'ils parlent somali et sont acculturés. Ils ont fondé la Somali African Muki Organisation (SAMO) afin de défendre leurs intérêts fonciers et leurs vies face aux chefs de guerre et aux Shabaab. Ainsi, parmi les réfugiés qui ont gagné le Kenya en 2011, comptaiton de nombreux agriculteurs chassés de leurs exploitations par les chefs de guerre qui avaient accaparé la terre ${ }^{14}$.

7 Marcel Djama dans "The Political Anthropology of "Pastoral Democracy" : Scope and Limitations in Somali History " (pp. 105-115) reprend la question des clans comme Lee Cassanelli, "Speculations on the Historical Origins of the "Total Somali Genealogy" ») (pp. 51-66) et Luca Ciabarri, "Trade, Lineages, Inequalities: Twists in the Northern Path to Modernity (pp. 67-85). Contrairement à ce qui est parfois affirmé, jamais Lewis n'a voulu construire de modèle, mais comme le montrent les trois auteurs, ses 
travaux fondateurs analysent la situation du Somaliland, une colonie «oubliée " à la veille du départ des colons. La résistance à l'ordre colonial s'est incarnée dans les généalogies et dans les contrats territoriaux liant les groupes prêts à payer le prix du sang (mag). Toutefois, le développement exponentiel (après 1980) des ventes du bétail pour nourrir les pèlerins du hajj en Arabie saoudite a bouleversé les sociétés et les modes de production agricoles tant au Nord qu'au Sud, tourné vers l'exportation de coton et de banane. D'une certaine façon, l'ex-Somaliland a pris sa revanche aux dépens de l'ex-Somalie italienne plus peuplée et marquée par l'agro-industrie héritée de la colonisation. Devenue la «boucherie de La Mecque » après avoir été «boucherie » des Britanniques d'Aden, la Somalie est désormais soumise à l'entrée en force de l'Arabie dans la péninsule, également par le biais de la réforme de l'islam.

8 L'islam somalien, s'il sous-tend la totalité des contributions, apparaît plus précisément dans « Political Islam in Somali History » de Hussein M. Adam (pp. 117-135), «Women, Islamists and the Military Regime in Somalia: The New Family Law and Its Implications » d'Abdurahman M. Abdullahi (Baadiyow) (pp. 137-160) et dans les textes, consacrés à la possession, de Marja Tiilikainen, "Spirits and the Human World in Northern Somalia » (pp. 161-184) et d'Anita Adam, «A Saar Gaamuri in Somalia : Spirit Possession as Expression of Women's Autonomy?» (pp. 185-204). Tous les auteurs notent que l'islam somali, marqué par le soufisme, la musique, les danses et le culte des saints auprès desquels s'installent ses fidèles dans des jamacaa (on dirait zaouïa au Maghreb), est fortement concurrencé, et même dénoncé, par les partisans d'un islam rigoriste. Après le putsch de Siyaad Barre (1969), la défaite en Ogadén (1978) et le déclenchement de la guerre civile, des milliers de Somaliens gagnèrent, soit la péninsule Arabique et le Golfe, soit les camps de Djibouti, d'Éthiopie, du Kenya et du Yémen, soit l'Europe et les États-Unis. Soumise à des prêches radicaux tant au MoyenOrient qu'en Occident, la diaspora somalienne concourt largement à la diffusion de l'islamisme. Les hostilités commencèrent, il y a quarante ans, quand siyaad Barre imposa l'écriture du somali avec des caractères latins « impies » et un code de la famille assurant l'égalité entre hommes et femmes en matière d'héritage : il fit fusiller les aw (cheikh) qui menaient l'opposition. Toutefois, son régime affaibli dut en passer par les volontés saoudiennes qui lui envoyèrent des prêcheurs wahhabites qui imposèrent l'arabe, le vêtement, la barbe, la séparation des sexes dans l'espace public et se déchaînèrent contre les cultes "féminins" des saar (zar). Dommage, d'ailleurs, qu'A. Adam ait omis de citer M. Leiris qui a décrit, avec Abba Jérôme dans L'Afrique fantôme, les zar à Gondär! Au Somaliland, de prospères cliniques islamiques, dirigées par des hommes, s'efforcent de les évincer du marché de la guérison des traumatismes de la guerre civile. On pourrait ajouter que cet islam réformé, qui combat pour rétablir le califat et l'unité de l'Umma, se heurte à une forte résistance : les jeunes de la diaspora, recrutés par les Shabaab, s'engagent surtout pour défendre la Somalie ${ }^{15}$.

9 L'histoire, enfin, est directement traitée par le témoignage de John Drysdale, «Reflections 1943-63 » (pp. 17-33) et par les contributions de Gérard Prunier, «Benign Neglect versus La Grande Somalia : the Colonial Legacy and the Postcolonial Somali State " (pp. 35-49), de Sally Healey, "Reflections on the Somali State: What Went Wrong and Why it might not Matter» (pp. 367-384) et Charles Geshekter, «The Social Anthropologist as Historian: Ioan Lewis as Chronicler of Somalia» (pp. 385-402). J. Drysdale est un témoin précieux et rare car son histoire personnelle coïncide depuis 1941 avec celle de la péninsule somali. Il commanda en Birmanie, pendant la Seconde Guerre mondiale, un contingent de Somalilandais ${ }^{16}$, fut en poste au Somaliland, quand 
Lewis commença ses études, et occupa diverses fonctions diplomatiques en Somalie indépendante. Il rappelle combien les Somalilandais eurent le sentiment d'avoir été trahis par la Grande-Bretagne lorsqu'elle rétrocéda le Hawd à l'Éthiopie. Ce témoignage, tout précieux qu'il soit, doit être replacé dans son contexte historique. En 1934, la frontière du Somaliland et de l'Éthiopie avait été bornée sur le terrain et elle fut longtemps la seule, dans la Corne, à être ainsi fixée. Selon M. Djama et John Markakis, toute arbitraire qu'elle soit, cette limite tracée au cordeau a été ratifiée par le temps et l'usage. La frontière, rupture de charge, procure des emplois formels et informels et protège les pâturages du Hawd utilisés par les Issaq, partisans du Mouvement national somalien (MNS) séparatiste, des appétits des Ogaadeeni qui soutenaient Siyaad ${ }^{17}$.

Les autres contributions rappellent, arguments à l'appui, combien le dessein colonial fut différent au Somaliland et en Somalia. Pour les Britanniques, ce n'était qu'un point d'appui comme le fut Djibouti pour la France, l'Érythrée pour l'Italie ou les présides marocains pour l'Espagne. La Somalie italienne fut, avec la Lybie, une colonie qui devait fournir la métropole en matières premières et offrir des terres aux colons. Sous l'AFIS, elle devait démontrer l'excellence du projet colonial italien qui, débarrassé de ses oripeaux fascistes, devait fonder un État. Les Somalilandais, même indépendants, se sont passés d'un État où ils étaient minoritaires en continuant d'utiliser les liens claniques et généalogiques plus qu'au Sud où les clans d'agriculteurs Digil et Mirifle fondaient le parti XDMS afin de s'affirmer face aux grandes fédérations claniques. Tous s'accordent à reconnaître que l'œuvre de Lewis forme une chronique irremplaçable du lent affaissement de l'État somalien tel qu'il l'a remarquablement synthétisé dans Understanding Somalia and Somaliland.

11 Un regret, pour terminer, Nurrudin Farah ${ }^{18}$ n'est nulle part mentionné dans cet ouvrage alors que Said S. Samatar auteur de "A Nation's Literary Death Tops Its Political Demise " (pp. 205-220) et Boobe Yusuf Duale, "Cabdillahi Suddaan Maxamed Timocadde ${ }^{19}$ (1920-1973) : The Man with the Roaring Voice, Looking like a Lion» (pp. 259-277), interviennent à propos de la poésie et de la littérature. Ils expliquent combien les poètes somaliens ont perdu le statut de porte-parole de la nation avec la création de l'État et sa dérive dictatoriale pourtant dénoncée par N. Farah dans ses romans et études. Peut-être lui reproche-t-on d'utiliser l'anglais, compris hors de Somalie, et de laver, en conséquence, le linge sale clanique au vu et au su des étrangers? En effet, il aborde sans détour les sujets les plus brûlants, les plus tabous: les relations avec l'Éthiopie dans Maps et entre les Somaliens dans Secrets, la place des femmes dans la société, sujet de From a Crooked Rib, de Gifts et leur exclusion du politique dans Yesterday, Tomorrow - Voices of the Somali Diaspora. Imagine-t-on un livre sur l'Égypte où Naguib Mahfouz, Sonallah Ibrahim, Alaa El-Aswany n'apparaîtraient nulle part?

12 En conservant leur liberté de ton et leur esprit critique, les contributeurs de ce livre ont rendu le plus sincère des hommages à l'œuvre fondatrice de Lewis. Cet ouvrage est le livre de chevet de ceux qui s'intéressent à la Somalie et cherche à comprendre les Somaliens et les Somali. Il vaut la peine d'être lu et relu. 


\section{NOTES}

7. De 1950 à 1960, l'Amministrazione Fiduciara Italiana della Somalia (AFIS) préparait, sous les auspices de l'ONU, l'indépendance de l'ex-colonie sous la tutelle de l'exmétropole.

8. Somalien désigne les ressortissants de la république de Somalie, somali, la langue, et Somali, ses locuteurs à Djibouti, en Éthiopie et au Kenya.

9. Avec A. Y. FARAH, « Making Peace in Somaliland», M. DJAMA et A. GASCON (dir.), «La Corne dans tous ses États », Cahiers d'Études africaines, XXXVII (2), 146, 1997, pp. 349-378.

10. Understanding Somalia and Somaliland, Culture, History, Society, London, Hurst \& Company, 2008 (voir mon compte-rendu, Cahiers d'Études africaines, 205, 2012, pp. 283-287).

11. À l'INALCO, la répétitrice de somali (1988-1990) m'enseigna l'unité de la langue comme un fait établi. Le répétiteur d'amharique (1971-1974) m'avait assuré qu'on n'y trouvait aucun dialecte alors qu'un de mes anciens élèves de la campagne d'alphabétisation (Zämacha 1975) en poste au Gojjam avait remarqué le contraire.

12. Voir le site Ethnologue. Pour la localisation, voir les cartes Michelin $n^{\circ} 745$ et KnowHow Reisen.

13. Paris, Arguments, 1995.

14. G. SCHLEE, "Conflict Resolution and Reconciliation as a Component of the Improvement of Farming Systems Project (IFSP) », in W. G. C. SMIDT \& K. ABRAHAM (eds.), Discussing Conflict in Ethiopia, Zürich-Berlin, Lit Verlag, 2007, pp. 119-144.

15. H. OUAKNINE, Al Shabaab et la diaspora somalienne de Londres. Fonctionnement d'un réseau de recrutement islamiste et représentation de la jeunesse immigrée, Mémoire de Master 2, Paris, Institut français de géopolitique, Université Paris 8, 2010.

16. Le Bataillon somali, recruté par les Français, s'illustra sur le front lors de la Première Guerre mondiale.

17. J. MARKAKIS, «The Ishaq Ogaden Dispute », in A. HJORT AJ ORNÄS\&M. A. MOHAMMED SALIH (eds.), Ecology and Politics, Uppsala, Nordiska Afrikainstitutet, 1989, pp. 157-169.

18. Découvert en France par Jacqueline Bardolph qui a traduit ses romans les plus remarquables.

19. En somali, Timocadde : aux cheveux blancs. 